\title{
EGFL7-blocking antibody inhibit pituitary adenoma proliferation and invasion
}

\begin{abstract}
Objective: To study the effect of EGFL7-blocking monoclonal antibody inhibiting the proliferation and invasion of mouse adrenocorticotropic hormone (ACTH)-secreting pituitary adenomas (ACTHomas) AtT-20 cells.
\end{abstract}

Methods: After treated with different concentration of EGFL7-blocking monoclonal antibody for 24, 48, $72 \mathrm{~h}$, the proliferation of AtT-20 cells was determined by MTS. Simultaneously, ACTH secretion of AtT-20 cells by ELISA and the mRNA expression of invasion related genes such as E-cadherin, snail, MMP-2 and MMP-9 by Real-time PCR were detected.

Results: EGFL7-blocking monoclonal antibody could effectively inhibit the proliferation and ACTH secretion of AtT-20 cells in a dose-dependent and time-dependent manner. At the same time, EGFL7-blocking monoclonal antibody significant down-regulated the mRNA level of snail, MMP-2 and MMP-9 in AtT-20 cells.

Conclusion: EGFL7-blocking monoclonal antibody could inhibit mouse pituitary adenoma AtT-20 cells proliferation and invasion.

Keywords: EGFL7, monoclonal antibody, AtT-20 cells, proliferation, invasion
Volume I 2 Issue I - 2022

\author{
Qian Liu, Jianpeng Wang, Hua Gao, Yazhuo \\ Zhang \\ Beijing Neurosurgical Institute, Beijing Tiantan Hospital, China
}

Correspondence: Yazhuo Zhang, Beijing Neurosurgical Institute, Beijing Tiantan Hospital, Capital Medical University, Beijing 100050, China, Email zyz2004520@yeah.net

Received: January 27, 2022 | Published: February 14, 2022
Abbreviations: EGFL7, Epidermal growth factor-like domain 7; GH, growth hormone; PRL, prolactin; ACTH, adrenocorticotropic hormone; ACTHomas, adrenocorticotropic hormone (ACTH)secreting pituitary adenomas; GHomas, GH-secreting pituitary adenoma; CNS, central nervous system

\section{Introduction}

Pituitary adenoma are one of the most common nervous system tumors. Most pituitary adenomas have the function of secreting distinct pituitary hormones such as growth hormone $(\mathrm{GH})$, prolactin (PRL) and adrenocorticotropic hormone (ACTH). ${ }^{1}$ Cushing's disease accounts for $5 \%-10 \%$ of hormone secreting pituitary adenomas, due to ACTH-secreting pituitary adenomas (ACTHomas) leads to hypercortisolemia. However, the mechanism of ACTHomas is still unclear. ${ }^{2}$ The pituitary adenoma invasion is an important factor associated with the clinical prognosis. Endothelial cell derivedsecreted factor epidermal growth factor-like domain 7 (EGFL7), plays an important role in angiogenesis. ${ }^{3}$ Previously, we reported that increased EGFL7 expression is correlated with the clinical progression, poor prognosis, and tumor grade in hormone-producing pituitary adenomas. ${ }^{4-6}$

However, the effect of EGFL7 in ACTHomas remains to be completely elucidated. Therefore, the present study aimed to investigate the effect of inhibition of EGFL 7 on proliferation and ACTH secretion in mouse ACTHomas AtT-20 cells. In vitro administration of anti-EGFL7 antibodies could effectively inhibit the proliferation and ACTH secretion of AtT-20 cells in a dose-dependent and timedependent manner. At the same time, EGFL7-blocking monoclonal antibody significant down-regulated the mRNA level of E-cadherin, snail and vimentin in AtT-20 cells. Taken together, these findings suggest that EGFL7 might serve as a potential novel biomarker for ACTHomas.

\section{Material and methods}

\section{Cell culture}

Mouse ACTHomas AtT-20 cells were purchased from ATCC and cultured in F12 medium (containing 2.5\% fetal bovine serum, 15\% horse serum, 100 units $/ \mathrm{mL}$ penicillin, 100 units $/ \mathrm{mL}$ streptomycin), cultured in an incubator containing $5 \%$ carbon dioxide at $37{ }^{\circ} \mathrm{C}$ for 48 hours. Once every 72 hours replaced the culture medium.

\section{Cell proliferation assay}

AtT-20 cells in logarithmic growth stage were placed in 96-well microplates, after 24 hours of culture, various concentrations of EGFL 7 monoclonal antibodies were added, and the final concentrations were $(10,50$ and $100 \mu \mathrm{g} / \mathrm{mL})$. The normal human IgG was used as a control. Each concentration was parallel to 3 wells, and the treatment was continued for 24,48 and 72 hours. $20 \mu \mathrm{L}$ MTS solution was added to each well with $100 \mu \mathrm{L}$ culture medium to culture at $37^{\circ} \mathrm{C}$ for $4 \mathrm{~h}$. The absorbance value at the wavelength of $490 \mathrm{~nm}$ was measured under the microplate reader.

\section{ELISA assay}

AtT-20 cells in logarithmic growth stage were placed in 96-well microplates, after 24 hours of culture, various concentrations of EGFL7 monoclonal antibodies were added. The supernatant of AtT20 cells treated with 10,50 and $100 \mu \mathrm{g} / \mathrm{mL}$ monoclonal antibody was stored at $-80^{\circ} \mathrm{C}$ for ELISA to detect the level of active ACTH using ACTH RapidBio ELISA kit, which performed as described previously. ${ }^{6}$

\section{qRT-PCR}

Total RNA was extracted from frozen tumor samples and normal anterior pituitaries using Trizol. Firststrand cDNA synthesis was 
generated using the kit according to the manufacturer's instructions. qRT-PCR primer sets are listed in Table 1.

Table I RT-PCR primer list

Gene name Forward sequence $\left(5^{\prime}-3^{\prime}\right)$ Reverse sequence $\left(5^{\prime}-3^{\prime}\right)$

E-cadherin TAGAGGGTCACCGCGTC GGGCTGGAGTCTGAACT

Snail CAATCGGAAGCCTAACTA CAGATGAGCATTGGCAGCG

MMP-2 AGACATACATCTTTGCTG CTTGAAGAAGTAGCTGTG

MMP-9 CCTGGAGACCTGAGAACCAAT CTTGAAGAAGTAGCTGTG

GAPDH GGTGGTCTCCTCTGACTTCAA

CCAAATTCGTTGTCATACCAG

\section{Statistical analysis}

SPSS 13.0 statistical software was used for data analysis. The data are expressed as mean \pm standard deviation. The differences between groups were analyzed by two independent sample t-test and variance (ANOVA). The value $\mathrm{p}<0.05$ was considered statistically significant.

\section{Results}

\section{Anti-EGFL7 antibody inhibited the proliferation of AtT-20 cells}

We previously demonstrated that attenuation of EGFL7 expression inhibits the growth of hormone-producing pituitary adenomas progression and invasion. ${ }^{4,5}$ Therefore, to further evaluate the effects and therapeutic potential of inhibition of EGFL7 in ACTHomas, MTS experiments were performed with a commercially available antibody that specifically binds to the EGFL7 protein. As shown in Figure 1, in ACTHomas cells, treatment with increasing concentrations of the anti-EGFL7 blocking antibody led to significant decreases in cell proliferation compared with normal IgG control in a time-dependent manner.

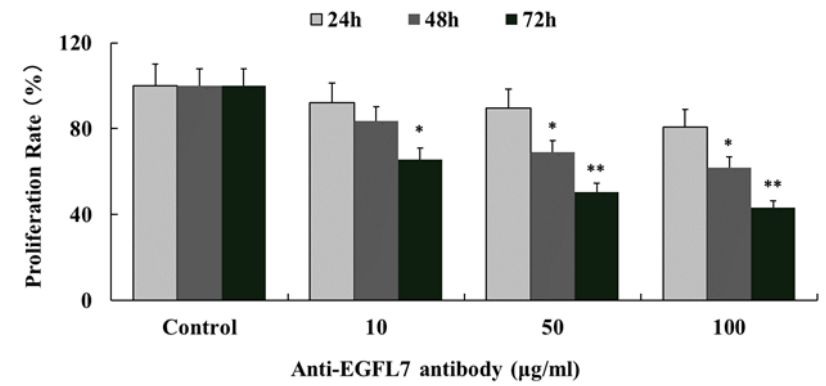

Figure I Anti-EGFL7 antibody inhibited the proliferation of AtT-20 cells by MTS. $* P<0.05, * * p<0.01$ versus normal human IgG control.

\section{EGFL7-blocking antibody inhibited the ACTH secretion from AtT-20 cells}

Furthermore, the levels of secreted ACTH in the culture supernatant from AtT-20 cells were also assayed by ELISA using an antibody against the EGFL7 protein. The most representative results after treatment with EGFL7-blocking antibody for $72 \mathrm{~h}$ are shown in Figure 2. Treatment of AtT-20 cells with 50 and $100 \mu \mathrm{g} /$ $\mathrm{mL}$ anti-EGFL7 antibodies for $72 \mathrm{~h}$ significantly reduced the level of secreted ACTH as compared with normal human $\operatorname{IgG}$ control. The serum ACTH levels were reduced to $120 \pm 25,89 \pm 19$, and $74 \pm 13 \mathrm{pg} /$ $\mathrm{mL}$ in response to 10,50 , and $100 \mu \mathrm{g} / \mathrm{mL}$ of anti-EGFL 7 antibodies, respectively (Figure 2$)(\mathrm{p}<0.01)$.

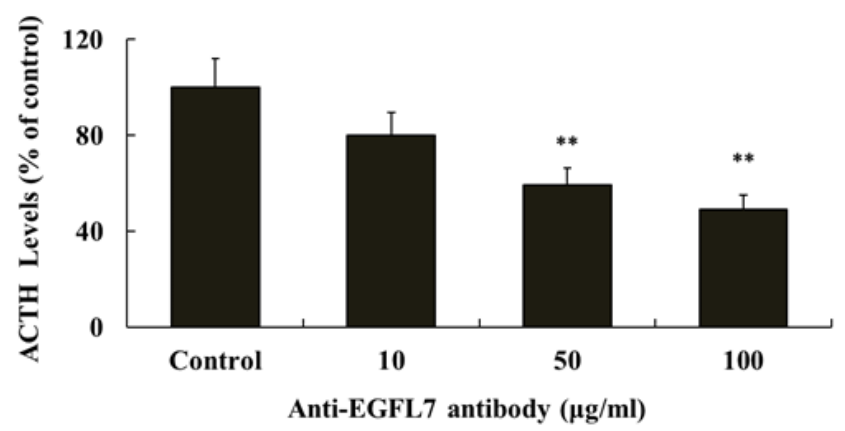

Figure 2 Downregulation of EGFL7 inhibited the ACTH secretion from AtT20 cells by ELISA. ${ }^{*} \mathrm{p}<0.0$ I versus normal human IgG.

\section{EGFL7-blocking antibody down-regulated the mRNA level of invasion related genes in AtT-20 cells}

In order to determine whether EGFL7 monoclonal antibody affects the invasion of AtT-20 cells, we detected the effect of EGFL7 monoclonal antibody on the expression of invasion related genes of AtT-20 cells by qRT-PCR. Treatment of AtT-20 cells with 10 and 50 $\mu \mathrm{g} / \mathrm{mL}$ anti-EGFL7 antibodies for $24 \mathrm{~h}$, without affecting the cell proliferation activity, significantly inhibited the mRNA expression levels of snail, MMP-2 and MMP-9 and induced the mRNA expression levels of E-cadherin $(\mathrm{p}<0.01)$ (Figure 3$)$.

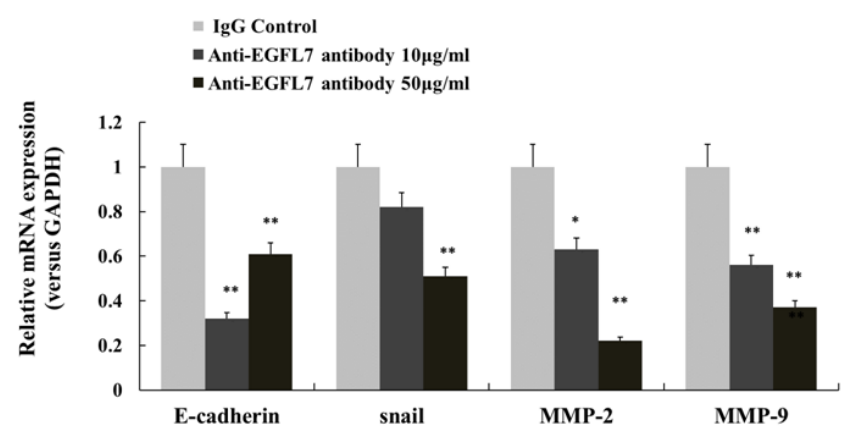

Figure 3 Anti-EGFL7 antibody inhibited the invasion of AtT-20 cells by RTPCR. $* \mathrm{P}<0.05, * * \mathrm{p}<0.0$ I versus normal human IgG control.

\section{Discussion}

Tumor arise in anterior pituitary gland is pituitary adenoma, which are the most common central nervous system tumor. ${ }^{8}$ Most pituitary adenomas are functional in nature, which secreting PRL, GH or ACTH. ${ }^{9-11}$ In our group, for the first time we found that, compared to non-invasive GH-secreting pituitary adenoma (GHomas), the expression of EGFL7 protein in invasive GHomas was markedly increased. The expression of EGFL7 was significantly associated with the histologic subtypes, tumor volume, invasiveness and recurrence of GHomas via Notch2/DLL3 signaling pathway. ${ }^{4,5}$ Furthermore, our published data ${ }^{5}$ indicated knockdown of EGFL7 expression suppresses GH3 and GT1-1 cells proliferation and invasion in vitro and inhibits human GHomas growth in vivo. The data suggest that as a Notch agonist, EGFL7 may potentially be an appropriate novel molecular target for future development of GHomas medical therapy.

In this study, our finding suggests that EGFL7 may contribute to the regulation of proliferation and ACTH secretion in mouse ACTHomas AtT-20 cells. Furthermore, anti-EGFL7 antibodies inhibit the mRNA expression levels of invasion related genes snail and MMP-2/MMP-9 without affecting the cell proliferation activity. 
Taken together, EGFL7 may serve as a potential novel therapeutic target for ACTHomas.

\section{Conclusion}

EGFL7-blocking monoclonal antibody could inhibit mouse pituitary adenoma AtT-20 cells proliferation and invasion. These findings raised the possibility that EGFL7 might serve as a useful biomarker to assess ACTHomas invasion and prognosis or a potential therapeutic target for ACTHomas treatment.

\section{Acknowledgments}

This work was supported by the Beijing Municipal Natural Science Foundation (7192033), Beijing Hospitals Authority Youth Programme (QML20200502), National Natural Science Foundation of China (81502154).

\section{Conflicts of interest}

The authors declare that they have no competing interests.

\section{References}

1. Mehta GU, Lonser RR, Mehta GU, et al. Management of hormonesecreting pituitary adenomas. Neuro Oncol. 2017;19(6):762-773.

2. Fukuoka H, Shichi H, Yamamoto M, et al. The Mechanisms Underlying Autonomous Adrenocorticotropic Hormone Secretion in Cushing's Disease. Int J Mol Sci. 2020 Nov 30;21(23):9132.
3. Parker LH, Schmidt M, Jin SW, et al. The endothelial-cell-derived secreted factor Egfl7regulates vascular tube formation. Nature, 2004;428(6984):754-758.

4. Liu Q, Wang J, Yang H, et al. Attenuation of EGFL7 expression inhibits growth hormone-producing pituitary adenomas growth and invasion. Hum Gene Ther (in press).

5. Liu Q, Zhang J, Gao H, et al. Role of EGFL7/EGFR-signaling pathway in migration and invasion of growth hormone-producing pituitary adenomas. Sci China Life Sci. 2018;61(8):893-901.

6. Qian Liu, Taoyang Yuan, Hua Gao, et al. Anti-EGFL7 antibodies inhibit rat prolactinoma MMQ cells proliferation and PRL secretion. Open Chem. 2018;16:621-626.

7. Zhenye Li, Qian Liu, Chuzhong Li, et al. The role of TGF- $\beta / \mathrm{Smad}$ signaling in dopamine agonist-resistant prolactinomas. Molecular and Cellular Endocrinology. 2015;402:64-71.

8. Samridhi Banskota, David C. Adamson Pituitary Adenomas: From Diagnosis to Therapeutics. Biomedicines. 2021; 9(5):494.

9. Pernicone PJ, Scheithauer BW, Sebo TJ, et al. Pituitary carcinoma: a clinicopathologic study of 15 cases. Cancer. 1997;79:804-812.

10. Amy S Joehlin-Price, Douglas A Hardesty, Christina A, et al. Case report: ACTH-secreting pituitary carcinoma metastatic to the liver in a patient with a history of atypical pituitary adenoma and Cushing's disease. Diagn Pathol. 2017;12:34.

11. Scheithauer BW, Fereidooni F, Horvath E, et al. Pituitary carcinoma: an ultrastructural study of eleven cases. Ultrastruct Pathol. 2001;25(3):227242 . 